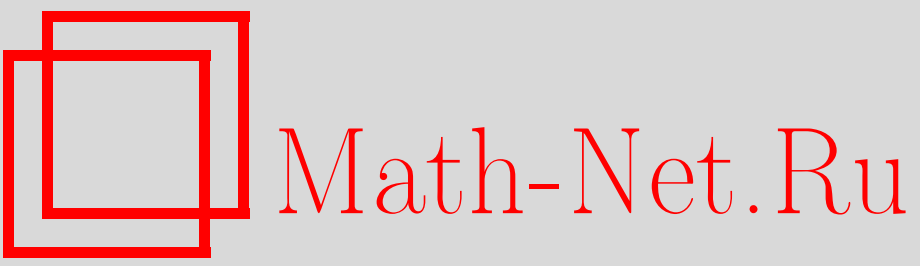

О. В. Баева, С. А. Павлова, О периодических решениях математической модели «политика-экономика», Итоги науки и техн. Сер. Соврем. мат. и ее прил. Tемат. обз., 2020, том 185, 28-36

DOI: https://doi.org/10.36535/0233-6723-2020-185-28-36

Использование Общероссийского математического портала Math-Net.Ru подразумевает, что вы прочитали и согласны с пользовательским соглашением

http: //www.mathnet.ru/rus/agreement

Параметры загрузки:

IP: 54.237 .59 .107

26 апреля 2023 г., 13:41:14 


\title{
О ПЕРИОДИЧЕСКИХ РЕШЕНИЯХ МАТЕМАТИЧЕСКОЙ МОДЕЛИ «ПОЛИТИКА-ЭКОНОМИКА»
}

\author{
(c) 2020 г. \\ О. В. БАЕВА, С. А. ПАВЛОВА
}

\begin{abstract}
АннотАция. Рассматривается математическая модель «политика-экономика», описываемая нелинейной системой дифференциальных уравнений с параметром. Определены условия существования ненулевого положительного периодического решения.
\end{abstract}

Ключевъе слова: математическая модель, нелинейная система дифференциальных уравнений с параметром, ненулевое периодическое решение.

\section{ON PERIODIC SOLUTIONS OF THE MATHEMATICAL MODEL "POLITICS-ECONOMICS"}

\author{
(c) 2020 O. V. BAEVA, S. A. PAVLOVA
}

\begin{abstract}
In this paper, we consider a mathematical model "politics-economy" described by a nonlinear system of differential equations with a parameter and obtain conditions for the existence of a nonzero positive periodic solution.
\end{abstract}

Keywords and phrases: mathematical model, nonlinear system of differential equations with a parameter, nonzero periodic solution.

AMS Subject Classification: 34K06, 34K10, 34K13

1. Введение. В основе предлагаемой модели лежит схема описания общества, принадлежащая Т. Парсонсу (см. [1]). Парсонс выделяет следующие подсистемы социальной системы: социетальное общество, систему поддержания институционализированных этнических образцов, экономическую и политическую системы. Динамику изменения данных подсистем опишем системой дифференциальных уравнений.

В качестве управляющего параметра возьмем уровень пассионарного напряжения (характеристика этноса). Пассионарное напряжение - это качественная характеристика, которую следует рассматривать как некую усредненную оценку этнической системы.

Политическую систему будем описывать степенью политической дифференциации (количеством политических институтов) $G(t)$, экономическую систему - количеством единиц фондов $E(t)$, социетальное сообщество - количеством социальных институтов $K(t)$, посредством которых происходит интеграция общества, а систему поддержания институционализированных этнических образцов - количеством социальных институтов $D(t)$, отвечающих за поддержание институционализированных этнических образцов.

Развитие политической системы опишем дифференциальным уравнением

$$
\frac{d G}{d t}=G_{G}+G_{E}+G_{K}
$$

слагаемые в правой части уравнения имеют следующий смысл: 
(i) Слагаемое $G_{G}=k_{G G}\left(e^{\delta \lambda-\delta_{1}}-1\right) G$ описывает процесс самоорганизации политической системы, который обеспечивает поддержание и развитие общественного строя. Он включает действия власти, препятствующие изменению существующей политической системы. Эти действия могут выражаться в принятии законов о разграничении властных полномочий. Здесь $k_{G G}\left(e^{\delta \lambda-\delta_{1}}-1\right)$ - учет пассионарности $\lambda$ при «построении» и поддержке государства, множитель $\left(e^{\delta \lambda-\delta_{1}}-1\right)$ - относительный уровень пассионарности, множитель $k_{G G}$ - положительный коэффициент.

(ii) Слагаемое $G_{E}=\left(l_{G}(\varepsilon)-k_{G E}(t, \nu) E\right) E$ представляет усилия людей в момент времени $t$ по укреплению политического режима за счет средств экономики. Степень этих усилий определяется условиями жизни, т.е. уровнем развитости экономики. Изменение политической системы зависит от состояния экономики. Увеличение уровня развития экономической системы $(E)$ не приводит к постоянному усложнению политической системы $(G)$. Наступает момент «насыщения», при котором увеличение экономических вложений во власть не приводит к дальнейшему «росту» политической системы. Здесь $\left(l_{G}(\varepsilon)-k_{G E}(t, \nu) E\right)$ - доля капитала в момент времени $t$, направленного на укрепление политического режима, $\nu=(\lambda, \varepsilon)-$ параметр, изменение которого влияет на развитие системы.

(iii) Слагаемое $G_{K}=k_{G K}(\lambda)(K+D) G$ - ограничения на скорость изменения политической системы, связанные с действующими в обществе традицией (нравственностью) и нормативным порядком. Эти ограничения сказываются на высоком уровне «социализации» $(K+D)$. Сюда входят затраты политической системы на поддержание нормативного порядка и этнических образцов. Также это борьба с преступлениями против порядка и против общества. Затраты на борьбу приводят к упрощению политической системы, к централизации и сосредоточению власти. Чем активнее государство борется с противоправными действиями, тем более централизованной и монополизированной становится власть.

Развитие экономической системы описывается дифференциальным уравнением

$$
\frac{d E}{d t}=E_{E}-E_{G}-E_{K}
$$

смысл слагаемых в правой части таков:

(i) Слагаемое $E_{E}=k_{E E}\left(e^{\delta \lambda-\delta_{1}}-1\right) E-$ усилия людей по развитию экономики. Это процесс воспроизводства экономических ресурсов. Экономическая система пополняет запас ресурсов за счет собственных вложений;

(ii) Слагаемое $E_{G}=\left(l_{E}(\varepsilon)-k_{E G}(t, \nu) G\right) G$ - экономические вливания во властные структуры в момент времени $t$. Для поддержания и развития политической системы требуется вложение экономических ресурсов. Слагаемое $E_{G}$ - некоторая «саморегуляция» воздействия политической системы на экономическую. Смысл квадратичной зависимости в том, что это воздействие определяется коллективным состоянием системы и выражается числом возможных взаимодействий социальных институтов;

(iii) Слагаемое $E_{K}=k_{E K}(\lambda)(K+D) E$ - ограничивающие факторы, связанные с этнокультурными традициями и нормативным порядком. С увеличением уровня «социализации» $(K+D)$ возрастает ее сдерживающее воздействие на развитие экономики. Чем выше уровень традиции $(D)$ и нормативного порядка $(K)$, тем большие ограничения они оказывают на рост экономической системы.

Любое общество рождается не на пустом месте, а на обломках когда-то сплоченных государств. Поэтому в качестве начальных данных $\left(\left.G\right|_{t=0}=G_{0},\left.E\right|_{t=0}=E_{0}\right)$ мы можем выбрать соответствующие величины предшествующих социальных систем (константы $G_{0}, K_{0}$ ).

Предположим, что социетальное сообщество $(K)$ и система поддержания институционализированных этнических образцов $(D)$ зафиксированы на некотором уровне и не меняются со временем. 
Рассмотрим систему дифференциальных уравнений

$$
\begin{cases}\frac{d G}{d t}=k_{G G}\left(e^{\delta \lambda-\delta_{1}}\right) G+\left(l_{G}(\varepsilon) E-k_{G E}(t, \nu) E^{2}\right)-k_{G K}(\lambda)(K+D) G, & \left.G\right|_{t=0}=G_{0}, \\ \frac{d E}{d t}=k_{E E}\left(e^{\delta \lambda-\delta_{1}}\right) E+\left(l_{E}(\varepsilon) G-k_{E G}(t, \nu) G^{2}\right)-k_{E K}(\lambda)(K+D) E, & \left.E\right|_{t=0}=E_{0} .\end{cases}
$$

Для упрощения исследования предположим, что интенсивность реакции политических институтов $k_{G} G$ и $k_{E} E$ интенсивность развития экономики равны. Предположим также, что равны доли социальных институтов $(K$ и $D)$, влияющих на политическую дифференциацию и экономический рост, т.е. $k_{G} K=k_{E} K$.

Пусть

$$
\begin{gathered}
f(\lambda)=k_{G G}\left(e^{\delta \lambda-\delta_{1}}-1\right)-k_{G K}(\lambda)(K+D), \\
k_{1}(t, \lambda, \varepsilon)=k_{G E}(t, \nu), \quad k_{2}(t, \lambda, \varepsilon)=k_{E G}(t, \nu), \quad l_{G}(\varepsilon)=l_{E}(\varepsilon)=l(\varepsilon), \quad \nu=(\lambda, \varepsilon), \\
x=G, \quad y=E, \quad x_{0}=G_{0}, \quad y_{0}=E_{0} .
\end{gathered}
$$

Тогда система примет вид

$$
\begin{cases}\dot{x}=f(\lambda) x+l(\varepsilon) y-k_{1}(t, \lambda, \varepsilon) y^{2}, & \left.x\right|_{t=0}=x_{0}, \\ \dot{y}=-l(\varepsilon) x+f(\lambda) y+k_{1}(t, \lambda, \varepsilon) x^{2}, & \left.y\right|_{t=0}=y_{0} .\end{cases}
$$

где $x$-уровень политической дифференциации $G, y$-развитие экономической системы $E$.

Определим условия, при которых развитие системы будет представлять собой циклический процесс.

Переходя к полярным координатам $x=\rho \cos \varphi, y=\rho \sin \varphi$, получим систему вида

$$
\left\{\begin{array}{l}
\dot{\rho}=(A(\lambda)+X(t, \varphi, \rho, \lambda, \varepsilon)) \rho, \\
\dot{\varphi}=\mu(\varepsilon)+\Phi(t, \varphi, \rho, \lambda, \varepsilon),
\end{array}\right.
$$

где

$$
\begin{gathered}
A(\lambda)=f(\lambda), \quad \mu(\varepsilon)=-l(\varepsilon), \\
X(t, \varphi, \rho, \lambda, \varepsilon)=-k_{1}(t, \lambda, \varepsilon) \rho \cos \varphi \sin ^{2} \varphi+k_{2}(t, \lambda, \varepsilon) \rho \cos ^{2} \varphi \sin \varphi, \\
\Phi(t, \varphi, \rho, \lambda, \varepsilon)=k_{2}(t, \lambda, \varepsilon) \rho \cos ^{3} \varphi+k_{1}(t, \lambda, \varepsilon) \rho \sin ^{3} \varphi, \\
\mu(0)=0, \quad X(t, \varphi, 0, \lambda, \varepsilon)=0, \quad \Phi(t, \varphi, 0, \lambda, \varepsilon)=0 .
\end{gathered}
$$

Предположим, что для вектор-функции $\mu(\varepsilon)$ справедливо представление

$$
\mu(\varepsilon)=B \varepsilon+\Theta(\varepsilon),
$$

где $\mu(0)=0$. Полагаем, что вектор-функция $\gamma(\lambda)=f(\lambda)$ представляется в виде

$$
\gamma(\lambda)=D \lambda+o(|\lambda|), \quad \lambda \rightarrow 0 .
$$

Непосредственно можно убедиться, что для системы (2) при введенных предположениях выполнены следующие условия:

(a) $\rho \in \mathbb{R}^{m}, \varphi \in \mathbb{R}^{p}, \varepsilon \in \mathbb{R}^{l}, \lambda \in \mathbb{R}^{q}$, где $\mathbb{R}^{s}$ - s-мерное векторное пространство;

(b) $\mu(\varepsilon)-p$-мерная вектор-функция, определенная и непрерывная на множестве

$$
E\left(\delta_{0}\right)=\left\{\varepsilon \in \mathbb{R}^{l}:|\varepsilon| \leqslant \delta_{0}\right\} ;
$$

(c) $\Phi(t, \varphi, \rho, \lambda, \varepsilon)$ - p-мерная вектор-функция, определенная и непрерывная по совокупности переменных, $T$-периодическая по $t$ на множестве

$$
\begin{gathered}
\mathrm{M}\left(\delta_{0}\right)=[0, T] \times \mathbb{R}^{p} \times \mathrm{M}_{0}\left(\delta_{0}\right) \times \Lambda\left(\delta_{0}\right) \times E\left(\delta_{0}\right), \\
\mathrm{M}_{0}\left(\delta_{0}\right)=\left\{x \in \mathbb{R}^{m}:|x| \leqslant \delta_{0}\right\}, \quad \Lambda\left(\delta_{0}\right)=\left\{\lambda \in \mathbb{R}^{q}:|\lambda| \leqslant \delta_{0}\right\},
\end{gathered}
$$

$T>0, \delta_{0}>0$ - некоторые постоянные числа; 
(d) на множестве $\mathrm{M}\left(\delta_{0}\right)$ справедливо неравенство

$$
\left\|\Phi\left(t, \varphi^{\prime}, x, \lambda^{\prime}, \varepsilon^{\prime}\right)-\Phi\left(t, \varphi^{\prime \prime}, x, \lambda^{\prime \prime}, \varepsilon^{\prime \prime}\right)\right\| \leqslant c_{1}\left(\delta_{0}\right) \cdot\left|\varphi^{\prime}-\varphi^{\prime \prime}\right|+c_{2}\left(\delta_{0}\right)\left|\lambda^{\prime}-\lambda^{\prime \prime}\right|+c_{3}\left(\delta_{0}\right)\left|\varepsilon^{\prime}-\varepsilon^{\prime \prime}\right|,
$$

где $c_{1}\left(\delta_{0}\right) \rightarrow 0, c_{2}\left(\delta_{0}\right) \rightarrow 0, c_{3}\left(\delta_{0}\right) \rightarrow 0$ при $\delta_{0} \rightarrow 0$, причем $\Phi(t, \varphi, 0, \nu)=0, \lim _{x \rightarrow 0} \Phi(t, \varphi, x, \lambda, \varepsilon)=0$ равномерно относительно $t, \phi, \lambda, \varepsilon$ на множестве $[0, T] \times \mathbb{R}^{p} \times \mathrm{M}_{0}\left(\delta_{0}\right) \times \Lambda\left(\delta_{0}\right) \times E\left(\delta_{0}\right)$;

(е) для всякого числа $\delta \in\left(0, \delta_{0}\right]$ на множестве $E\left(\delta_{0}\right)$ справедливо равенство

$$
\mu(\varepsilon)=B \varepsilon+\Theta(\varepsilon),
$$

где $B-(p \times l)$-матрица, вектор-функция $\Theta(\varepsilon)$ удовлетворяет условию Липшица по $\varepsilon$ с постоянной $\tau(\delta), \tau(\delta) \rightarrow 0$ при $\delta \rightarrow 0$;

(f) $A(\lambda)-(m \times m)$-матрица, определенная и непрерывная на множестве $\Lambda\left(\delta_{0}\right)$;

(g) $X(t, \varphi, x, \lambda, \varepsilon)-(m \times m)$-матрица, определенная и непрерывная по совокупности переменных, $T$-периодическая по $t$ на множестве $\mathrm{M}\left(\delta_{0}\right)$;

(h) на множестве $\mathrm{M}\left(\delta_{0}\right)$ справедливы неравенства

$$
\begin{gathered}
\|A(\lambda)\| \leqslant R, \quad\|X(t, \varphi, x, \lambda, \varepsilon)\| \leqslant L, \\
\left\|X\left(t, \varphi^{\prime}, x, \lambda^{\prime}, \varepsilon^{\prime}\right)-X\left(t, \varphi^{\prime \prime}, x, \lambda^{\prime \prime}, \varepsilon^{\prime \prime}\right)\right\| \leqslant l_{1}\left(\delta_{0}\right) \cdot\left|\varphi^{\prime}-\varphi^{\prime \prime}\right|+l_{2}\left(\delta_{0}\right)\left|\lambda^{\prime}-\lambda^{\prime \prime}\right|+l_{3}\left(\delta_{0}\right)\left|\varepsilon^{\prime}-\varepsilon^{\prime \prime}\right|,
\end{gathered}
$$

где $l_{1}\left(\delta_{0}\right) \rightarrow 0, l_{2}\left(\delta_{0}\right) \rightarrow 0, l_{3}\left(\delta_{0}\right) \rightarrow 0$ при $\delta_{0} \rightarrow 0$;

$$
\left\|A\left(\lambda^{\prime}\right)-A\left(\lambda^{\prime \prime}\right)\right\| \leqslant r\left(\delta_{0}\right)\left|\lambda^{\prime}-\lambda^{\prime \prime}\right|
$$

$r\left(\delta_{0}\right) \rightarrow 0$ при $\delta_{0} \rightarrow 0 ; X(t, \varphi, 0, \lambda, \varepsilon)=0, \lim _{x \rightarrow 0} X(t, \varphi, x, \lambda, \varepsilon)=0$ равномерно относительно $t, \phi, \lambda, \varepsilon$ на множестве $[0, T] \times \mathbb{R}^{p} \times \mathrm{M}_{0}\left(\delta_{0}\right) \times \Lambda\left(\delta_{0}\right) \times E\left(\delta_{0}\right)$.

Пусть $C\left(d_{0}, k\right)$ - класс $T$-периодических по $t$ вектор-функций $F(t)$, определенных на сегменте $[0, T]$, удовлетворяющих условиям

$$
\|F(t)\| \leqslant d_{0}, \quad\left\|F\left(t^{\prime}\right)-F\left(t^{\prime \prime}\right)\right\| \leqslant k\left|t^{\prime}-t^{\prime \prime}\right|
$$

для любых $t^{\prime}, t^{\prime \prime} \in[0, T]$, где $d_{0}, k$ - некоторые положительные числа.

В основе доказательства теоремы о существовании ненулевого периодического решения системы (2) лежит теорема [2].

Теорема 1. Пусть выполнены следующие условия.

1. К и $\Lambda$-замкнутые компактные множества некоторых линейных нормированных пространств, $K$ - выпуклое множество;

2. на подмножестве множества $K \times \Lambda$ определен такой оператор $T_{\lambda}$, что для любого $x \in K$ существует единственное $\lambda \in \Lambda$, удовлетворяющее включению $T_{\lambda} x \in K$;

3. если $x_{n} \rightarrow x_{0}, \lambda_{n} \rightarrow \lambda_{0}, y_{n}=T_{\lambda_{n}} x_{n}, y_{n} \rightarrow y_{0}$, mo $y_{0}=T_{\lambda_{0}} x_{0}$.

Тогда существуют такие $x^{*} \in K, \lambda^{*} \in \Lambda$, что $x^{*}=T_{\lambda^{*}} x^{*}$.

2. Основные результаты. Сформулируем основной результат работы. Задача о существовании $T$-периодического решения системы (2) сводится к задаче определения условий существования таких чисел $d, \delta_{0}$, что для любой вектор-функции $F(t) \in C(d, k)$ найдется вектор $\lambda \in \Lambda\left(\delta_{0}\right)$, удовлетворяющий условию

$$
\left(Y_{F}\left(T, \varphi_{T}, \lambda, \varepsilon\right)-E\right) \cdot \alpha=0 .
$$

Здесь $Y_{F}\left(t, \varphi_{t}, \lambda, \varepsilon\right)$ - фундаментальная матрица решений системы

$$
\dot{y}=\left[A(\lambda)+X\left(t, \varphi_{t}, F(t), \lambda, \varepsilon\right)\right] y,
$$

удовлетворяющая условию $Y_{F}\left(0, \varphi_{t}, \lambda, \varepsilon\right)=E$, где $E$ - единичная матрица.

Решение системы (3) определяется равенством

$$
y_{F}\left(t, \varphi_{t}, \lambda, \varepsilon\right)=Y_{F}\left(t, \varphi_{t}, \lambda, \varepsilon\right) \cdot \alpha,
$$


где $y_{F}\left(0, \varphi_{t}, \lambda, \varepsilon\right)=\alpha, \alpha$ - некоторый постоянный вектор, принадлежащий множеству $W(r)=$ $\{\alpha:|\alpha| \leqslant r\}$. Равенство (4) при любом фиксированном $\lambda \in \Lambda\left(\delta_{0}\right)$ на множестве $C(d, k)$ определяет оператор $\Gamma(\lambda) F(t) \rightarrow y_{F}\left(t, \varphi_{t}, \lambda, \varepsilon\right)$, который любой вектор-функции $F(t) \in C(d, k)$ ставит в соответствие вектор-функцию $y_{F}\left(t, \varphi_{t}, \lambda, \varepsilon\right)$.

Теорема 2. Неподвижные точки оператора $\Gamma(\lambda)$ являются T-периодическими решениями системы дифференциальных уравнений (2), принадлежащими множеству $C(d, k)$.

Доказательство. Пусть при некотором $\lambda \in \Lambda\left(\delta_{0}\right)$ вектор-функция $\rho \in C(d, k)$ - неподвижная точка оператора $\Gamma(\lambda)$. Это значит, что при любом $t \in[0, T]$ справедливо соотношение $\rho(t)=Y_{\rho}\left(t, \varphi_{t}, \lambda, \varepsilon\right) \cdot \alpha$, т.е. при $\rho=F(t)$ вектор-функция $\rho$ является решением системы $(3)$, а следовательно, решением системы (2). Теорема доказана.

Теорема 3. Пусть выполнены следующие условия:

(1) система уравнений (2) удовлетворяет условиям (a)-(h);

(2) в условии (d) $\operatorname{rang} B=p$;

(3) вектор-функиия $\gamma(\lambda)=\left(\gamma_{1}(\lambda), \gamma_{2}(\lambda), \ldots, \gamma_{s}(\lambda)\right)$ представима в виде $\gamma(\lambda)=D \lambda+o(|\lambda|)$, где $D-(s \times q)$-матрица, вектор-функиия о $(|\lambda|)$ удовлетворяет условию Липшица по $\lambda$ с постоянной $p(\delta), p(\delta) \rightarrow 0$ при $\delta \rightarrow 0 ;$

(4) $\operatorname{rang} D=s$.

Тогда существуют такие число $d>0$ и значение параметра $\nu^{*}=\left(\lambda^{*}, \varepsilon^{*}\right)$, что система $(2)$ имеет ненулевое T-периодическое решение из класса $C(d, k)$.

Доказательство. Рассмотрим второе уравнение в системе (2). Для того чтобы решение

$$
\varphi_{t}=\varphi^{F}\left(t, \varphi_{0}, \lambda, \varepsilon\right)
$$

системы (2) было T-периодическим, необходимо и достаточно, чтобы выполнялось равенство

$$
\mu(\varepsilon) T+\int_{0}^{T} \Phi\left(t, \varphi_{t}, F(t), \lambda, \varepsilon\right) d t=0 .
$$

Согласно условию (d) и условию 2 теоремы, равенство (5) примет вид

$$
\varepsilon_{1}=-B_{1}^{-1}\left(B_{2} \varepsilon_{2}+\frac{1}{T} \int_{0}^{T}\left[\Phi\left(t, \varphi_{t}, F(t), \lambda, \varepsilon\right)+\Theta(\varepsilon)\right] d t\right),
$$

где $B_{1}$ - неособенная $(p \times p)$-матрица, $B_{2}-(p \times(l-p))$-матрица, $\varepsilon=\operatorname{colon}\left(\varepsilon_{1}, \varepsilon_{2}\right), \varepsilon_{1}-p$-мерный вектор, $\varepsilon_{2}-(l-p)$-мерный вектор.

Тогда по [3, теорема 1] существуют такие числа $d>0, \delta_{1}>0$, что для любой векторфункции $F(t) \in C(d, k)$ и любого фиксированного $\lambda \in \Lambda\left(\delta_{0}\right)$ существует значение параметра $\varepsilon=\left(\varepsilon_{1}, \varepsilon_{2}\right) \in E\left(\delta_{1}\right)$, где $\varepsilon_{1}$ - единственная неподвижная точка оператора, определенного равенством (6) для фиксированного $\varepsilon_{2}$, при котором решение $\varphi_{t}=\varphi^{F}\left(t, \varphi_{0}, \lambda, \varepsilon\right)$ системы (2) является T-периодическим.

Таким образом, определена вектор-функция $\varepsilon=\Psi(F, \lambda)$, являющаяся единственной неподвижной точкой оператора $\Gamma$ при фиксированном $\lambda$, т.е. $Г \varepsilon=\varepsilon$. При замене $\varepsilon$ будет меняться $\lambda$. Следовательно, $\varepsilon-$ функция от $\lambda$.

Докажем, что $\varepsilon(\lambda)$ удовлетворяет условию Липшица по переменной $\lambda$. Действительно,

$$
\begin{aligned}
\left|\varepsilon\left(\lambda_{1}\right)-\varepsilon\left(\lambda_{2}\right)\right|= & \left|\Gamma_{F}\left(\lambda_{1}\right) \varepsilon\left(\lambda_{1}\right)-\Gamma_{F}\left(\lambda_{2}\right) \varepsilon\left(\lambda_{2}\right)\right|= \\
& =\left|\Gamma_{F}\left(\lambda_{1}\right) \varepsilon\left(\lambda_{1}\right)-\Gamma_{F}\left(\lambda_{1}\right) \varepsilon\left(\lambda_{2}\right)+\Gamma_{F}\left(\lambda_{1}\right) \varepsilon\left(\lambda_{2}\right)-\Gamma_{F}\left(\lambda_{2}\right) \varepsilon\left(\lambda_{2}\right)\right| \leqslant \\
& \leqslant \eta_{1}\left|\varepsilon\left(\lambda_{1}\right)-\varepsilon\left(\lambda_{2}\right)\right|+\eta_{2}\left|\lambda_{1}-\lambda_{2}\right| .
\end{aligned}
$$


Отсюда получаем

$$
\left|\varepsilon\left(\lambda_{1}\right)-\varepsilon\left(\lambda_{2}\right)\right| \leqslant \frac{\eta_{2}}{1-\eta_{1}}\left|\lambda_{1}-\lambda_{2}\right|
$$

Фиксируем вектор-функцию $F(t) \in C(d, k)$. Подставив решение $\varphi_{t}=\varphi^{F}\left(t, \varphi_{0}, \lambda, \varepsilon\right)$, вектор-функцию $F(t) \in C(d, k)$ и вектор $\varepsilon(\lambda)$ в первое уравнение системы (2), получим систему (3), решением которой является вектор-функция $y_{F}\left(t, \varphi_{t}, \lambda, \varepsilon\right)$, определенная равенством (4). Для того чтобы вектор-функция $y_{F}\left(t, \varphi_{t}, \lambda, \varepsilon\right)$ была $T$-периодическим решением системы $(3)$, достаточно, чтобы вектор $\lambda$ удовлетворял равенству

$$
D \lambda+o(|\lambda|)+\phi_{F}\left(T, \varphi_{T}, \lambda, \varepsilon(\lambda)\right)=0 .
$$

Так как по условию теоремы $\operatorname{rang} D=s$, то для простоты рассуждений будем считать, что матрицу $D$ можно представить в виде $D=\left[\Delta_{1}, \Delta_{2}\right]$, det $\Delta_{1} \neq 0$. Матрица $D$ имеет размер $(s \times q)$, $\Delta_{1}-(s \times s), \Delta_{2}-(s \times(q-s))$.

Пусть $\lambda=\operatorname{colon}\left(\lambda_{1}, \lambda_{2}\right)$, где $\lambda_{1}-s$-мерный вектор, $\lambda_{2}-(s-q)$-мерный вектор. Тогда уравнение (7) примет вид

$$
\Delta_{1} \lambda_{1}+\Delta_{2} \lambda_{2}+o(|\lambda|)+\phi_{F}\left(T, \varphi_{T}, \lambda, \varepsilon(\lambda)\right)=0
$$

Так как матрица $\Delta_{1}$ неособенная, то уравнение $(7)$ можно представить в виде

$$
\lambda_{1}=\Delta_{1}^{-1}\left(\Delta_{2} \lambda_{2}+o(|\lambda|)+\phi_{F}\left(T, \varphi_{T}, \lambda, \varepsilon(\lambda)\right)\right) .
$$

Определим оператор $S$ равенством

$$
S \lambda_{1}=\Delta_{1}^{-1}\left(\Delta_{2} \lambda_{2}+o(|\lambda|)+\phi_{F}\left(T, \varphi_{T}, \lambda, \varepsilon(\lambda)\right)\right) .
$$

Докажем, что оператор $S$ имеет единственную неподвижную точку, т.е. существует единственный вектор $\lambda_{1}$, для которого $S \lambda_{1}=\lambda_{1}$. Так как

$$
\lim _{\lambda \rightarrow 0} \frac{o(|\lambda|)}{|\lambda|}=0
$$

то выберем такое число $\delta_{1}>0$, что при всех $|\lambda| \leqslant \delta_{1}$ выполняется неравенство

$$
\|o(|\lambda|)\| \leqslant \frac{\delta_{1}}{3\left\|\Delta_{1}^{-1}\right\|} .
$$

Тогда для любого $\lambda$, удовлетворяющего условию $|\lambda| \leqslant \delta_{1}$, выполняется неравенство

$$
\left\|\Delta_{1}^{-1} o(|\lambda|)\right\| \leqslant \frac{\delta_{1}}{3}
$$

Так как $\lim _{\lambda_{2} \rightarrow 0}\left(\Delta_{2} \lambda_{2}\right)=0$, то существует такое число $\delta^{\prime}$, что $\left|\lambda_{2}\right| \leqslant \delta^{\prime} \leqslant \delta_{1}$ и

$$
\left\|\Delta_{1}^{-1} \Delta_{2} \lambda_{2}\right\| \leqslant \frac{\delta_{1}}{3}
$$

Введем обозначение $\Lambda_{1}\left(\delta_{1}\right)=\left\{\lambda_{1}:\left|\lambda_{1}\right| \leqslant \delta_{1}\right\}$. Зафиксируем $\left|\lambda_{2}^{*}\right| \leqslant \delta_{1}$. Согласно условию

$$
\lim _{d \rightarrow 0} \beta_{F}^{l}\left(T, \varphi_{T}, \lambda, \varepsilon\right)=0
$$

равномерно относительно $\lambda$; тогда число $d$ выберем таким образом, что для любой вектор-функции $F(t) \in C(d, k)$ имеет место неравенство

$$
\left\|\Delta_{1}^{-1} \phi_{F}\left(T, \varphi_{T}, \lambda, \varepsilon(\lambda)\right)\right\| \leqslant \frac{\delta_{1}}{3} .
$$

Учитывая неравенства (10)-(12), получим, что для любых $\lambda_{1}$, удовлетворяющих условию $\left|\lambda_{1}\right| \leqslant \delta_{1}, F(t) \in C(d, k)$ и фиксированных $\left|\lambda_{2}^{*}\right| \leqslant \delta_{1}$ имеем

$$
\begin{aligned}
& \left\|S \lambda_{1}\right\|=\left\|\Delta_{1}^{-1}\left(\Delta_{2} \lambda_{2}+o(|\lambda|)+\phi_{F}\left(T, \varphi_{T}, \lambda, \varepsilon(\lambda)\right)\right)\right\| \leqslant \\
& \quad \leqslant\left\|\Delta_{1}^{-1} o(|\lambda|)\right\|+\left\|\Delta_{1}^{-1} \Delta_{2} \lambda_{2}\right\|+\left\|\Delta_{1}^{-1} \phi_{F}\left(T, \varphi_{T}, \lambda, \varepsilon(\lambda)\right)\right\| \leqslant \frac{\delta_{1}}{3}+\frac{\delta_{1}}{3}+\frac{\delta_{1}}{3}=\delta_{1} .
\end{aligned}
$$


Таким образом, для фиксированных $\left|\lambda_{2}^{*}\right| \leqslant \delta_{1}$ и для любой вектор-функции $F(t) \in C(d, k)$ оператор $S$ отображает множество $\Lambda_{1}\left(\delta_{1}\right)$ в себя.

Докажем, что оператор $S$ является сжимающим на множестве $\Lambda_{1}\left(\delta_{1}\right)$ при фиксированных $F(t) \in C(d, k)$ и $\left|\lambda_{2}^{*}\right| \leqslant \delta_{1}$. Возьмем произвольным образом $\lambda_{1}^{\prime}, \lambda_{1}^{\prime \prime} \in \Lambda_{1}\left(\delta_{1}\right)$. Пусть $\lambda^{\prime}=$ $\operatorname{colon}\left(\lambda_{1}^{\prime}, \lambda_{2}^{*}\right), \lambda^{\prime \prime}=\operatorname{colon}\left(\lambda_{1}^{\prime \prime}, \lambda_{2}^{*}\right)$. Согласно условию 3 теоремы и [3, лемма 2] на множестве $\Lambda_{1}\left(\delta_{1}\right)$ справедливы неравенства

$$
\left\|o\left(\left|\lambda^{\prime}\right|\right)-o\left(\left|\lambda^{\prime \prime}\right|\right)\right\| \leqslant p(\delta)\left|\lambda^{\prime}-\lambda^{\prime \prime}\right|
$$

и

$$
\begin{aligned}
\left\|\phi_{F}\left(T, \varphi_{T}, \lambda^{\prime}, \varepsilon\left(\lambda^{\prime}\right)\right)-\phi_{F}\left(T, \varphi_{T}, \lambda^{\prime \prime}, \varepsilon\left(\lambda^{\prime \prime}\right)\right)\right\| & \leqslant \\
\leqslant H_{1}(\delta)\left|\lambda^{\prime}-\lambda^{\prime \prime}\right|+H_{2}(\delta)\left|\varepsilon\left(\lambda^{\prime}\right)-\varepsilon\left(\lambda^{\prime \prime}\right)\right| & \leqslant H_{1}(\delta)\left|\lambda^{\prime}-\lambda^{\prime \prime}\right|+H_{2}(\delta) \frac{\eta_{2}}{1-\eta_{1}}\left|\lambda^{\prime}-\lambda^{\prime \prime}\right|= \\
& =\left(H_{1}(\delta)+H_{2}(\delta) \frac{\eta_{2}}{1-\eta_{1}}\right)\left|\lambda^{\prime}-\lambda^{\prime \prime}\right|=\bar{H}(\delta)\left|\lambda^{\prime}-\lambda^{\prime \prime}\right|,
\end{aligned}
$$

где $p(\delta) \rightarrow 0, \bar{H}(\delta) \rightarrow 0$ при $\delta \rightarrow 0$. Выберем число $\delta_{1}$ таким образом, чтобы для любого $\delta \in\left(0, \delta_{1}\right]$ имело место неравенство

$$
\left\|\Delta_{1}^{-1}\right\|(p(\delta)+\bar{H}(\delta)) \leqslant \beta<1 .
$$

Тогда для любых $\lambda_{1}^{\prime}, \lambda_{1}^{\prime \prime} \in \Lambda_{1}\left(\delta_{1}\right)$ при фиксированных $F(t) \in C(d, k)$ и $\left|\lambda_{2}^{*}\right| \leqslant \delta_{1}$ выполняется неравенство

$$
\left\|S \lambda_{1}^{\prime}-S \lambda_{1}^{\prime \prime}\right\| \leqslant\left\|\Delta_{1}^{-1}\right\|(p(\delta)+\bar{H}(\delta))\left|\lambda^{\prime}-\lambda^{\prime \prime}\right| \leqslant \beta\left|\lambda^{\prime}-\lambda^{\prime \prime}\right|=\beta\left|\lambda_{1}^{\prime}-\lambda_{1}^{\prime \prime}\right|
$$

где $\beta<1$. Таким образом, оператор $S$ при фиксированных $F(t) \in C(d, k)$ и $\left|\lambda_{2}^{*}\right| \leqslant \delta_{1}$ является сжимающим на множестве $\Lambda_{1}\left(\delta_{1}\right)$.

Таким образом, мы установили, что существуют такие числа $d>0$ и $\delta_{1}>0$, что для любой фиксированной вектор-функции $F(t) \in C(d, k)$ и для любого фиксированного $\left|\lambda_{2}^{*}\right| \leqslant \delta_{1}$ оператор $S$ переводит замкнутое ограниченное выпуклое множество $\Lambda_{1}\left(\delta_{1}\right)$ в себя и является сжимающим оператором. По теореме Банаха оператор $S$ имеет единственную неподвижную точку в множестве $\Lambda_{1}\left(\delta_{1}\right)$, являющуюся решением уравнения (9), т.е. существует единственный вектор $\tilde{\lambda}_{1},\left|\tilde{\lambda}_{1}\right| \leqslant \delta_{1}$, для которого $S \tilde{\lambda}_{1}=\tilde{\lambda}_{1}$. Это означает, что для любой вектор-функции $F(t) \in C(d, k)$ существует единственный вектор $\tilde{\lambda} \in \Lambda\left(\delta_{1}\right), \tilde{\lambda}=\left(\tilde{\lambda}_{1}, \tilde{\lambda}_{2}\right)$, где $\tilde{\lambda}_{1}$ - единственная неподвижная точка оператора $S$, координаты $\tilde{\lambda}_{2}$ - фиксированные, обладающий тем свойством, что $m$-й столбец матрицы $\left(Y_{F}\left(T, \varphi_{T}, \tilde{\lambda}, \varepsilon(\tilde{\lambda})\right)-E\right) \cdot Q_{F}\left(T, \varphi_{T}, \tilde{\lambda}, \varepsilon(\tilde{\lambda})\right)$ обращается в нуль.

Пусть $c-m$-мерный вектор, первые $m-1$ координат которого равны нулю, а $m$-я координата отлична от нуля. Тогда

$$
\left(Y_{F}\left(T, \varphi_{T}, \tilde{\lambda}, \varepsilon(\tilde{\lambda})\right)-E\right) \cdot Q_{F}\left(T, \varphi_{T}, \tilde{\lambda}, \varepsilon(\tilde{\lambda})\right) \cdot c=0 .
$$

Положив $\alpha=Q_{F}\left(T, \varphi_{T}, \tilde{\lambda}, \varepsilon(\tilde{\lambda})\right) \cdot c$, приходим к равенству

$$
\left(Y_{F}\left(T, \varphi_{T}, \tilde{\lambda}, \varepsilon(\tilde{\lambda})\right)-E\right) \cdot \alpha=0 .
$$

Следовательно,

$$
y_{F}\left(t, \varphi_{t}, \tilde{\lambda}, \varepsilon(\tilde{\lambda})\right)=Y_{F}\left(t, \varphi_{t}, \tilde{\lambda}, \varepsilon(\tilde{\lambda})\right) \cdot Q_{F}\left(T, \varphi_{T}, \tilde{\lambda}, \varepsilon(\tilde{\lambda})\right) \cdot c
$$

$-T$-периодическое решение системы $(3)$. Так как $c \neq 0$, то это решение является ненулевым. Кроме того, функция $y_{F}\left(t, \varphi_{t}, \tilde{\lambda}, \varepsilon(\tilde{\lambda})\right)$, где $t \in[0, T],\left|\varphi_{t}\right| \leqslant \delta_{0}, \tilde{\lambda} \in \Lambda\left(\delta_{1}\right)$, принадлежит множеству $C(d, k)$. Действительно,

$$
\begin{aligned}
\left|y_{F}\left(t, \varphi_{t}, \tilde{\lambda}, \varepsilon(\tilde{\lambda})\right)\right|=\left\|Y_{F}\left(t, \varphi_{t}, \tilde{\lambda}, \varepsilon(\tilde{\lambda})\right) \cdot Q_{F}\left(T, \varphi_{T}, \tilde{\lambda}, \varepsilon(\tilde{\lambda})\right) \cdot c\right\| & \leqslant \\
& \leqslant\left\|Y_{F}\left(t, \varphi_{t}, \tilde{\lambda}, \varepsilon(\tilde{\lambda})\right)\right\| \cdot\left\|Q_{F}\left(T, \varphi_{T}, \tilde{\lambda}, \varepsilon(\tilde{\lambda})\right)\right\| \cdot|c| .
\end{aligned}
$$


Поскольку величины $Y_{F}\left(t, \varphi_{t}, \lambda, \varepsilon\right), Q_{F}\left(T, \varphi_{T}, \lambda, \varepsilon\right)$ ограничены, то где

$$
\left\|Y_{F}\left(t, \varphi_{t}, \tilde{\lambda}, \varepsilon(\tilde{\lambda})\right) \cdot Q_{F}\left(T, \varphi_{T}, \tilde{\lambda}, \varepsilon(\tilde{\lambda})\right)\right\| \leqslant\left\|Y_{F}\left(t, \varphi_{t}, \tilde{\lambda}, \varepsilon(\tilde{\lambda})\right)\right\| \cdot\left\|Q_{F}\left(T, \varphi_{T}, \tilde{\lambda}, \varepsilon(\tilde{\lambda})\right)\right\|=P,
$$

Тогда

$$
P=\sup _{t \in[0, T]}\left\|Y_{F}\left(t, \varphi_{t}, \tilde{\lambda}, \varepsilon(\tilde{\lambda})\right)\right\| \cdot \sup _{|z| \leqslant 1}\left|Q_{F}\left(T, \varphi_{T}, \tilde{\lambda}, \varepsilon(\tilde{\lambda})\right) z\right| .
$$

Кроме того,

$$
\left|y_{F}\left(t, \varphi_{t}, \tilde{\lambda}, \varepsilon(\tilde{\lambda})\right)\right| \leqslant\left\|Y_{F}\left(t, \varphi_{t}, \tilde{\lambda}, \varepsilon(\tilde{\lambda})\right)\right\| \cdot\left\|Q_{F}\left(T, \varphi_{T}, \tilde{\lambda}, \varepsilon(\tilde{\lambda})\right)\right\| \cdot|c|<P c .
$$

$$
\left\|\dot{y}_{F}\left(t, \varphi_{t}, \tilde{\lambda}, \varepsilon(\tilde{\lambda})\right)\right\| \leqslant\left(\|A(\tilde{\lambda})\|+\left\|X\left(t, \varphi_{t}, F(t), \tilde{\lambda}, \varepsilon(\tilde{\lambda})\right)\right\|\right) \cdot\left\|y_{F}\left(t, \varphi_{t}, \tilde{\lambda}, \varepsilon(\tilde{\lambda})\right)\right\| \leqslant(R+L) D c .
$$

Следовательно, за счет выбора вектора $c$ можно добиться справедливости неравенств $P c \leqslant d$, $(R+L) P c \leqslant k$. По теореме Лагранжа о среднем значении имеем

$$
\left\|y_{F}\left(t^{\prime}, \varphi_{t}, \tilde{\lambda}, \varepsilon(\tilde{\lambda})\right)-y_{F}\left(t^{\prime \prime}, \varphi_{t}, \tilde{\lambda}, \varepsilon(\tilde{\lambda})\right)\right\| \leqslant\left\|\dot{y}_{F}\left(t, \varphi_{t}, \tilde{\lambda}, \varepsilon(\tilde{\lambda})\right)\right\| \cdot\left|t^{\prime}-t^{\prime \prime}\right| \leqslant k \cdot\left|t^{\prime}-t^{\prime \prime}\right|,
$$

что доказывает принадлежность функции $y_{F}\left(t, \varphi_{t}, \tilde{\lambda}, \varepsilon(\tilde{\lambda})\right)$ классу $C(d, k)$.

Матрица $X\left(t, \varphi_{t}, F(t), \tilde{\lambda}, \varepsilon(\tilde{\lambda})\right)$ непрерывна на множестве $C(d, k) \times \Lambda\left(\delta_{1}\right)$ равномерно относительно $t \in[0, T]$ и $\left|\varphi_{t}\right| \leqslant \delta_{0}$, матрица $A(\tilde{\lambda})$ непрерывна на множестве $\Lambda\left(\delta_{1}\right)$. Поэтому оператор $\Gamma(\lambda)$, определенный равенством (4), непрерывен на множестве $C(d, k) \times \Lambda\left(\delta_{1}\right)$ и при любых $(F(t), \lambda, \varepsilon) \in C(d, k) \times \Lambda\left(\delta_{1}\right)$ имеет место соотношение

$$
\Gamma(\lambda) F(t)=y_{F}\left(t, \varphi_{t}, \lambda, \varepsilon(\lambda)\right) .
$$

Таким образом, получаем, что существуют такие числа $d>0, \delta_{1}>0$, что на множестве $C(d, k) \times \Lambda\left(\delta_{1}\right)$ оператор $\Gamma(\lambda)$ непрерывен и для любой вектор-функции $F(t) \in C(d, k)$ существует единственный вектор $\tilde{\lambda} \in \Lambda\left(\delta_{1}\right)$, удовлетворяющий соотношению

$$
\Gamma(\tilde{\lambda}) F(t)=y_{F}\left(t, \varphi_{t}, \tilde{\lambda}, \varepsilon(\tilde{\lambda})\right) \in C(d, k) .
$$

Тогда по теореме 1 существуют такие вектор-функция $F^{*}(t) \in C(d, k)$ и вектор $\lambda^{*} \in \Lambda\left(\delta_{1}\right)$, что $F^{*}(t)$ - неподвижная точка оператора $\Gamma\left(\lambda^{*}\right)$, т.е. $F^{*}(t)=Y_{F^{*}}\left(t, \varphi_{t}, \lambda^{*}, \varepsilon\left(\lambda^{*}\right)\right) \cdot \alpha$. По теореме 2 , так как

$$
F^{*}(t)=Y_{F^{*}}\left(t, \varphi_{t}, \lambda^{*}, \varepsilon\left(\lambda^{*}\right)\right) \cdot \alpha, \quad \alpha=Q_{F^{*}}\left(T, \varphi_{T}, \lambda^{*}, \varepsilon\left(\lambda^{*}\right)\right) \cdot c \neq 0,
$$

то $F^{*}(t)$ - ненулевое $T$-периодическое решение системы (2) при $\lambda=\lambda^{*}$.

В итоге получаем, что существует такое значение параметра $\nu^{*}=\left(\lambda^{*}, \varepsilon^{*}\right)$, где $\varepsilon^{*}=\varepsilon\left(\lambda^{*}\right)$, что

$$
F^{*}(t)=Y_{F^{*}}\left(t, \varphi_{t}, \lambda^{*}, \varepsilon^{*}\right) \cdot Q_{F^{*}}\left(T, \varphi_{T}, \lambda^{*}, \varepsilon^{*}\right) \cdot c
$$

- $T$-периодическое решение системы дифференциальных уравнений $(2)$, т.е. $F^{*}(t)$-решение уравнения

при $\nu=\nu^{*}$. Теорема доказана.

$$
\dot{\rho}=[A(\lambda)+X(t, \varphi, \rho, \lambda, \varepsilon)] \rho
$$

Поскольку $\mu(\varepsilon)=-l(\varepsilon), A(\lambda)=f(\lambda)$, а $f(\lambda), l(\varepsilon)$ - скалярные величины, то $D, B$ - числа, отличные от нуля.

Так как $\operatorname{rang} B=1, \operatorname{rang} D=1$, то согласно теореме 3 существует единственное значение параметра $\nu=(\lambda, \varepsilon)$, при котором система $(2)$ (а следовательно, (1)) имеет ненулевое $T$-периодическое решение. Тем самым найдены условия, при которых получены периодические колебания в развитии политической и экономической систем. Это хорошо согласуется с политической и экономическими теориями (циклы реформ-контрреформ, экономические циклы Н. Кондратьева).

Докажем, что это решение положительно. За счет выбора числа $d>0$ решение $\varphi_{F}\left(t, \varphi_{0}, \lambda, \varepsilon\right)$ системы

$$
\dot{\varphi}=l(\varepsilon)+k_{2}(t, \lambda, \varepsilon) \rho \cos ^{3} \varphi+k_{1}(t, \lambda, \varepsilon) \rho \sin ^{3} \varphi
$$

с начальным значением $\varphi_{0}>0$ будет принадлежать промежутку $(0, \pi / 2)$, т.е. располагаться в первом квадранте. Поскольку матрица $A(\lambda)=f(\lambda)$ определена и непрерывна на множестве 
$\Lambda\left(\delta_{0}\right)$, то имеет место неравенство $l_{1} \leqslant f(\lambda) \leqslant l_{2}$. В [3] установлено, что для того, чтобы решение системы (2) было $T$-периодическим, необходимо выполнение равенства

$$
\left(\mathrm{P}_{F}\left(T, \varphi_{T}, \lambda, \varepsilon\right)-E\right) \cdot \alpha=\left(\mathrm{P}_{F}\left(T, \varphi_{T}, \lambda, \varepsilon\right)-E\right) \cdot Q_{F}\left(T, \varphi_{T}, \lambda, \varepsilon\right) c=0,
$$

в котором $\mathrm{P}_{F}\left(t, \varphi_{t}, \lambda, \varepsilon\right)$ - фундаментальная матрица решений системы

$$
\dot{\rho}=(A(\lambda)+X(t, \varphi, \rho, \lambda, \varepsilon)) \rho
$$

представимая в виде

$$
\mathrm{P}_{F}\left(T, \varphi_{T}, \lambda, \varepsilon\right)=\overline{\mathrm{P}}(T, \lambda)+H\left(T, \varphi_{T}, \lambda, \varepsilon\right)=e^{f(\lambda) T}+\beta^{F}\left(T, \varphi_{T}, \lambda, \varepsilon\right),
$$

где $\overline{\mathrm{P}}(t, \lambda)$ - фундаментальная матрица решений системы $\dot{\rho}=A(\lambda) \rho, \beta^{F}\left(T, \varphi_{T}, \lambda, \varepsilon\right)$ - элемент матрицы $H_{F}\left(T, \varphi_{T}, \lambda, \varepsilon\right)$, причем $\lim _{d \rightarrow 0} \beta^{F}\left(T, \varphi_{T}, \lambda, \varepsilon\right)=0$ равномерно относительно $\lambda$.

Определено, что $Q_{F}\left(T, \varphi_{T}, \lambda, \varepsilon\right)$ имеет вид $Q_{F}\left(T, \varphi_{T}, \lambda, \varepsilon\right)=1$, причем

$$
\operatorname{det} Q_{F}\left(T, \varphi_{T}, \lambda, \varepsilon\right)=1 \text {. }
$$

Выберем $c=c^{*}>0$. Тогда $\alpha=Q_{F}\left(T, \varphi_{T}, \lambda, \varepsilon\right) c=c^{*}$. В этом случае решение системы (2) определится равенством

$$
\rho_{F}\left(t, \varphi_{t}, \lambda, \varepsilon\right)=\mathrm{P}_{F}\left(t, \varphi_{t}, \lambda, \varepsilon\right) \alpha=e^{f(\lambda) t}+\beta^{F}\left(t, \varphi_{t}, \lambda, \varepsilon\right) .
$$

Приведем следующие оценки:

$$
\begin{gathered}
e^{l_{1} t} \leqslant e^{f(\lambda) t} \leqslant e^{l_{2} t}, \\
e^{l_{1} t}+\beta^{F}\left(t, \varphi_{t}, \lambda, \varepsilon\right) \leqslant e^{f(\lambda) t}+\beta^{F}\left(t, \varphi_{t}, \lambda, \varepsilon\right) \leqslant e^{l_{2} t}+\beta^{F}\left(t, \varphi_{t}, \lambda, \varepsilon\right), \\
\left(e^{l_{1} t}+\beta^{F}\left(t, \varphi_{t}, \lambda, \varepsilon\right)\right) c^{*} \leqslant e^{f(\lambda) t}+\beta^{F}\left(t, \varphi_{t}, \lambda, \varepsilon\right)^{*} \leqslant\left(e^{l_{2} t}+\beta^{F}\left(t, \varphi_{t}, \lambda, \varepsilon\right)\right) c^{*}, \\
\left(e^{l_{1} t}-\left|\beta^{F}\left(t, \varphi_{t}, \lambda, \varepsilon\right)\right|\right) c^{*} \leqslant\left|\left(e^{f(\lambda) t}+\beta^{F}\left(t, \varphi_{t}, \lambda, \varepsilon\right)\right) c^{*}\right| \leqslant\left(e^{l_{2} t}+\left|\beta^{F}\left(t, \varphi_{t}, \lambda, \varepsilon\right)\right|\right) c^{*} .
\end{gathered}
$$

За счет выбора числа $d>0$ можем добиться выполнения неравенства $\left|\beta^{F}\left(t, \varphi_{t}, \lambda, \varepsilon\right)\right|<e^{l_{1} t}$. Тогда

$$
\begin{aligned}
0<\left(e^{l_{1} t}-\left|\beta^{F}\left(t, \varphi_{t}, \lambda, \varepsilon\right)\right|\right) c^{*} \leqslant\left|\left(e^{f(\lambda) t}+\beta^{F}\left(t, \varphi_{t}, \lambda, \varepsilon\right)\right) c^{*}\right| & \\
& \leqslant\left(e^{l_{2} t}+\left|\beta^{F}\left(t, \varphi_{t}, \lambda, \varepsilon\right)\right|\right) c^{*}<e^{l_{2} t} c^{*}+e^{l_{1} t} c^{*},
\end{aligned}
$$

т.е. решение $\rho_{F}\left(t, \varphi_{t}, \lambda, \varepsilon\right)$ системы (2) положительно. Тем самым установлено, что решения системы (1) расположены в первом квадранте и являются положительными.

\section{СПИСОК ЛИТЕРАТУРЫ}

1. Гуц А. К., Коробицын В. В., Лаптев А. А., Паутова Л. А., Фролова Ю. В. Математические модели социальных систем. - Омск: Омск. гос. ун-т, 2000.

2. Терехин M. Т. Бифуркация периодических решений функционально-дифференциальных уравнений// Изв. вузов. Мат. - 1999. - 10 (449). - С. 37-42.

3. Терехин М. Т., Баева О. В. Существование ненулевых периодических решений у особого класса систем дифференциальных уравнений с параметром// Вестн. Рязанск. гос. ун-та им. С. А. Есенина. - 2007. $-3(16) .-$ C. $77-98$.

Баева Ольга Владимировна

Академия права и управления

Федеральной службы исполнения наказаний России, Рязань

E-mail: olga8682@mail.ru

Павлова Светлана Анатольевна

Академия права и управления

Федеральной службы исполнения наказаний России, Рязань 